\title{
Analyse du couplage pompe - circuit à partir d'un modèle Navier - Stokes instationnaire de l'écoulement dans la pompe
}

\author{
Coupling pump - circuit analysis using a Navier - \\ Stokes unsteady model for the flow in the pump
}

\author{
par F. Longatte $(*)$, G. Tatu (**) et J.-L. Kueny (*) \\ (*) Institut National Polytechnique de Grenoble, Laboratoire des Ecoulements Géophysiques \\ et Industriels de Grenoble \\ (**) Université Technologique et de Génie Civil de Bucarest
}

This article presents a numerical analysis of the coupling pump-circuit. This study takes into account simultaneously the interactions between mobile and fixed parts of a centrifugal pump, as well as the interactions between this same pump and the surrounding circuit. The first results show the necessity to take into account the circuit influence to quantify the field of pressure in the pump. In addition, the pump is a source of flow rate and pressure fluctuations in the circuit : these fluctuations depend strongly on the location of blades with respect to the volute tongue: the flow rate and the head are minimum when $a$ blade is placed just opposite to the volute tongue. Moreover, fluctuations intensities depend on the location of pump in the circuit.

\section{I — LA MODÉLISATION NUMÉRIQUE DES PHÉNOMÈNES INSTATIONNAIRES}

\subsection{Etude numérique de l'écoulement dans la pompe : interaction rotor-stator}

Pour prévoir les performances hydrauliques et mécaniques des pompes centrifuges, il est nécessaire de connaître précisément l'écoulement dans la roue et la volute de ces machines. Dans ce but, de nombreux travaux expérimentaux $[1,2,3]$ et numériques $[4,5,6,7,8]$ ont été réalisés. Notons cependant que la plupart des travaux numériques considèrent séparément l'écoulement dans la partie tournante et dans la partie fixe $[5,6]$ ou bien un écoulement potentiel ou quasi statique $[4,9]$. En fait, le comportement instantané des pompes centrifuges est le résultat de l'interaction entre le rotor et le stator. Aussi est-il nécessaire pour prévoir les performances de ces pompes de prendre en compte l'interaction roue-volute, donc de calculer simultanément l'écoulement instationnaire et turbulent dans les parties fixes et mobiles de la pompe. Nous avons opté pour le code de calcul industriel
N3S, développé à la Direction des Etudes et Recherches d'Electricité de France [10] et qui propose l'étude de l'écoulement tridimensionnel, visqueux, turbulent d'un fluide incompressible, par résolution des équations de Reynolds couplées au modèle de turbulence $k-\varepsilon$. La version instationnaire permet d'étudier l'écoulement dans des géométries présentant des domaines en rotation ou en translation par rapport aux autres domaines et permet ainsi de réaliser aussi bien des calculs dans des turbomachines présentant une roue et une volute ou un diffuseur aubé que des calculs dans plusieurs rangées de grilles d'aubes en mouvement.

La discrétisation en espace (fig. l) est basée sur la méthode des éléments finis de Galerkin avec une formulation mixte pour la pression statique et les autres grandeurs physiques (vitesse, $k$ et $\varepsilon$ ). Ainsi la première est approximée sur chaque élément (nœud P1), alors que les autres le sont sur chaque sous-élément (nœud P2).

1. Nœuds P1 et P2.

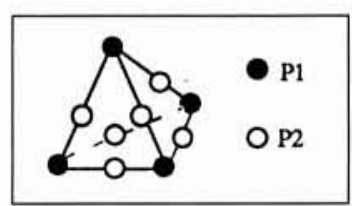


Pour la discrétisation temporelle, ce code fait appel à une méthode à pas fractionnaires. Chaque pas de temps est décomposé en une étape de convection, traitée par la méthode des caractéristiques, et en une étape de diffusion pour les grandeurs scalaires ou en un problème de Stokes pour le système couplé en vitesse-pression. Les systèmes linéaires sont résolus par un algorithme de gradient conjugué préconditionné par la diagonale et le problème de Stokes est résolu par l'algorithme d'Uzawa ou celui de Chorin.

Les conditions aux limites sont celles habituellement utilisées en mécanique des fluides : le profil de vitesse, l'énergie et la dissipation turbulente en entrée de domaine sont imposés, ainsi qu'une condition de sortie libre et une loi de paroi $(\kappa-\varepsilon)$. De plus, il faut fixer la vitesse de translation ou de rotation des différentes parties de la géométrie étudiée ainsi que le pas de temps.

\subsection{Etude de l'écoulement dans le circuit : propagation d'ondes planes}

Toute perturbation, produite dans n'importe quel endroit d'un circuit hydraulique, se propage sous la forme des ondes soniques, c'est-à-dire d'ondes se déplaçant à la vitesse du son. Quand les conditions de propagation changent, ces perturbations peuvent se refléter et se réfracter, en se superposant les unes aux autres et produire un régime instationnaire. Un cas particulier est celui présentant une perturbation périodique due à un composant du circuit, notamment les turbomachines. Cette perturbation est alors désignée sous le terme d'excitation. Pour prendre en compte le caractère ondulatoire des excitations du circuit, nous avons décidé de modéliser l'écoulement présent dans le circuit en considérant le fluide compressible, et le circuit unidimensionnel. Suivant ce modèle, la méthode des caractéristiques sous la forme des invariants de Riemann a été adoptée. La seule restriction provient du fait que cette méthode n'est valable que pour un fluide parfait. Nous pallions ce désavantage, en considérant des pertes de charges singulières en chaque point de discrétisation de la conduite, c'est-à-dire que chaque élément présentant une perte de charge singulière ou régulière est modélisé par un diaphragme [11] (fig. 2).

Pour chaque élément, il est nécessaire de connaître la pression en entrée et en sortie de l'élément ainsi que le débit transitant dans cet élément, soit trois inconnues. Or, en pratique, nous avons les deux équations de propagation d'ondes (onde directe et onde inverse) ainsi qu'une loi arbitraire reliant le débit dans l'élément et la différence de pression entre la sortie et l'entrée de ce même élément. Le problème est donc fermé. Il faut, avant tout calcul, définir les lois des différents organes ainsi qu'un pas de temps.

\subsection{Descriptif du couplage : interaction pompe-circuit}

Le but de cette étude est de connaître l'écoulement dans la pompe et le circuit environnant en ne fixant que les géomé- tries du circuit et de la pompe ainsi que la vitesse de rotation. En fait, tous les éléments du circuit sont connus (diaphragmes) sauf celui représentant la pompe ; cette dernière est modélisée à chaque pas de temps grâce au code N3S. Cependant, pour le calcul de la pompe, il est nécessaire de connaître le débit en entrée de roue. Pour le déterminer, nous couplons les deux codes de calcul grâce à un processus itératif : à chaque pas de temps, nous proposons un seul et même débit fictif pour le calcul dans le circuit et pour celui de la pompe et obtenons ainsi deux hauteurs d'élévations, celle fournie par le code du circuit et celle par le code N3S. Tant que ces deux hauteurs sont différentes, nous proposons un autre débit fictif (dichotomie), jusqu'à obtenir le débit réel donnant deux hauteurs d'élévation identiques. Toutefois, nous n'avons pu obtenir l'égalité parfaite entre ces deux hauteurs, nous avons considéré que le couplage était acceptable pour une différence pouvant aller de $0,1 \%$ à $1 \%$. Il est à noter que le couplage nécessite des précautions, notamment celle d'imposer pour les deux codes de calcul un même pas de temps suffisamment faible pour vérifier le nombre de courant des deux codes $\left({ }^{1}\right)$.

\section{II $\square$ CHOIX DE LA GÉOMÉTRIE ET DU CIRCUIT}

\subsection{Réalisation de la pompe et discrétisation}

Dans un premier temps, nous avons décidé d'étudier une géométrie bidimensionnelle de pompe centrifuge. Il nous fallait donc une roue ainsi qu'une volute bidimensionnelles. Nous avons choisi d'étudier une pompe ayant pour roue celle de la pompe de l'Université de Gênes [12] et pour volute, une volute en spirale logarithmique vérifiant la conservation du moment cinétique [13]. Les caractéristiques de la roue et de la volute sont présentées ci-dessous ainsi que la géométrie (tabl. 1, fig. 3).

Le maillage de la pompe (fig. 4) comporte 30000 nœuds $\mathrm{P} 2$. Le pas de temps $\Delta t_{\max }$ est fixé à $72 \mu \mathrm{s}\left(\mathrm{CFL}_{\mathrm{N} 3 \mathrm{~S}}<2\right)$; il faut donc 420 pas de temps pour avoir une rotation complète de la roue. La période de passage des aubes $t_{p}$ face au bec vaut 4,29 ms.

Cette géométrie est facilement modifiable et permettra d'étudier l'influence du jeu entre le bec de volute et les bords de fuite des aubes de la roue, ainsi que du nombre d'aubes.

(1) $C F L_{N 3 S}=\max \left(\frac{u \cdot \Delta t}{\Delta x}\right)<2$ et $C F L_{\text {circuit }}=\max \left(\frac{a \cdot \Delta t}{\Delta x}\right)<1$

avec $\Delta t$ pas de temps, $\Delta x$ taille d'une maille, $u$ vitesse du fluide et $a$ vitesse de propagation des ondes.

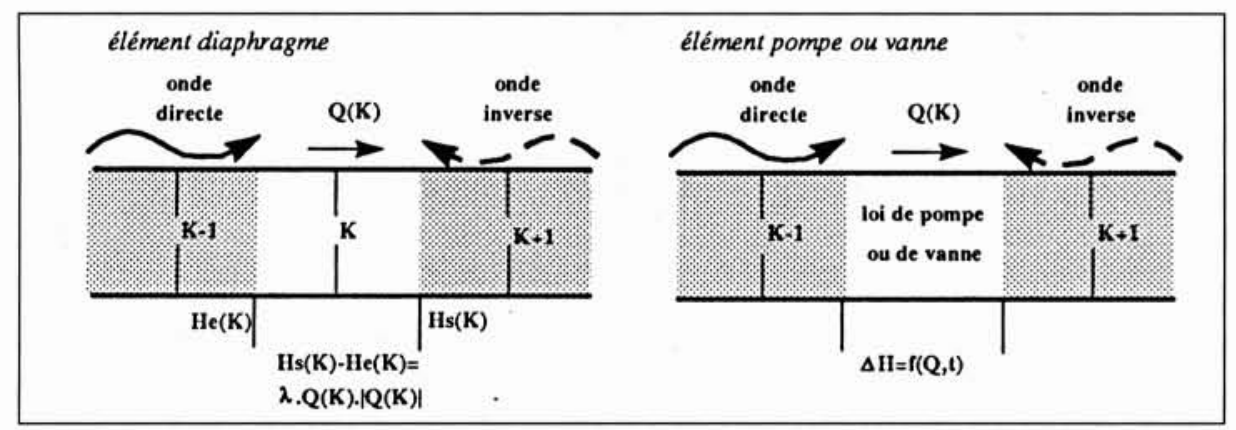

2. Modélisation de trois éléments consécutifs du circuit.
3 inconnues en $t+\Delta t$ : débit $\mathrm{Q}(\mathrm{K})$ pression en entrée $\mathrm{He}(\mathrm{K})$ pression en sortie $\mathrm{Hs}(\mathrm{K})$

2 équations :

onde directe

onde inverse

loi arbitraire :

loi de l'élément $K$. 
Tableau 1. - Caractéristiques de la pompe.

\begin{tabular}{|lc|cc|}
\hline \multicolumn{1}{|c|}{ débit $\boldsymbol{Q}_{\text {ref }}$} & $0,2894 \mathrm{~m}^{3} / \mathrm{s}$ & diamètre d'entrée de roue $\mathbf{2 .} \boldsymbol{R}_{\mathbf{1}}$ & $240 \mathrm{~mm}$ \\
\hline hauteur $\boldsymbol{H}_{\text {ref }}$ & $78,48 \mathrm{~m}$ & diamètre de sortie de roue $2 . \boldsymbol{R}_{\mathbf{2}}$ & $420 \mathrm{~mm}$ \\
\hline vitesse de rotation $\Omega$ & $2000 \mathrm{tr} / \mathrm{min}$ & épaisseur de la pompe $\boldsymbol{~}$ & $40 \mathrm{~mm}$ \\
\hline nombre d'aubes & 7 & angle de sortie du fluide $\alpha_{\mathbf{2}}$ & $16^{\circ}$ \\
\hline vitesse spécifique $\boldsymbol{N}_{\boldsymbol{s}}$ & 40 & position du bec (jeu $\left.=\boldsymbol{R}_{\text {bed }} / \boldsymbol{R}_{\mathbf{2}}-\mathbf{1}\right)$ & $3 \%$ \\
\hline
\end{tabular}

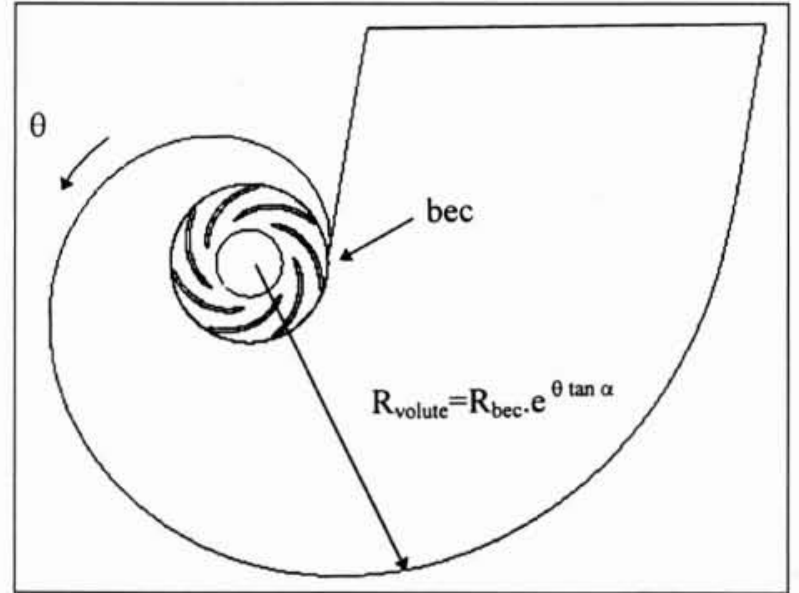

3. Géométrie étudiée 2D.

\subsection{Réalisation des deux circuits}

Afin de montrer l'influence du circuit sur le comportement de la pompe, nous avons réalisé deux circuits fermés symétriques de longueurs différentes. Du fait de la symétrie du problème, le næud milieu du circuit (nœud à mi-distance de l'aspiration et du refoulement de la pompe) ne peut qu'être un naud de pression. Les deux longueurs des circuits sont choisies afin que la pompe se trouve soit sur un naud de pression soit sur un ventre de pression, c'est-à-dire deux circuits présentant un temps de parcours $t_{c}\left({ }^{2}\right)$ des ondes de 2,0 et 2,5 fois la période de passage des aubes $t_{p}$. La vitesse du son $a$ étant imposée constante à $1000 \mathrm{~m} / \mathrm{s}$, les longueurs des circuits valent respectivement $8,57 \mathrm{~m}$ et $10,71 \mathrm{~m}$ pour un nœud et un ventre de pression au niveau de la pompe. Les deux circuits comportent 60 éléments identiques de diamètre $0,40 \mathrm{~m}$ et de perte de charge totale de $78,48 \mathrm{~m}$ pour un débit de $0,2894 \mathrm{~m}^{3} / \mathrm{s}$ (point de fonctionnement de la pompe étudiée).

\section{CALCULS RÉALISÉS}

Pour analyser et quantifier l'importance des phénomènes d'interaction pompe-circuit, il est intéressant de réaliser simultanément l'étude de la pompe sans le circuit et l'étude de cette même pompe avec son circuit environnant.

(2) $t_{c}=L a$ avec $L$ longueur du circuit et $a$ vitesse de propagation des ondes.

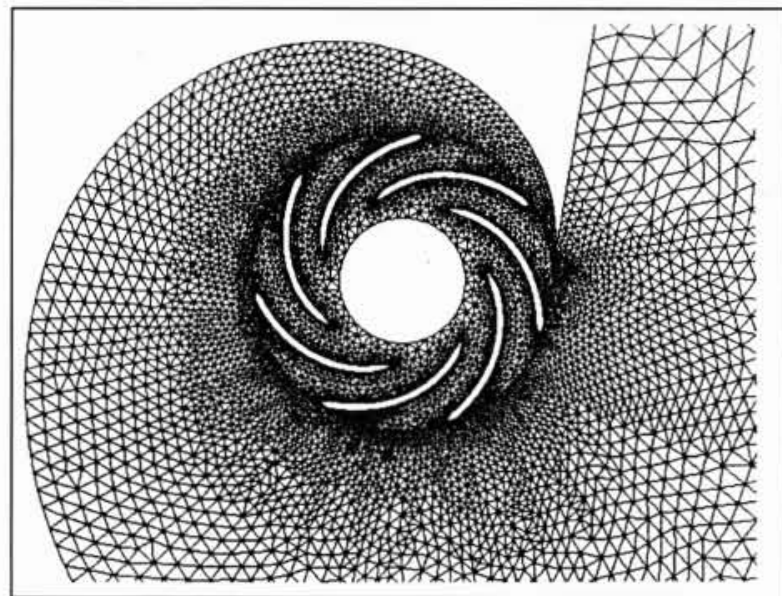

4. Aperçu du maillage de la roue.

- 3.1 Calcul de la pompe seule : calcul non couplé avec le
circuit

Un calcul de l'écoulement dans la pompe a été réalisé sans couplage avec le circuit pour le point de dessin de la roue, c'est-à-dire, pour un débit constant de $0,2894 \mathrm{~m}^{3} / \mathrm{s}$ et une vitesse de rotation de $2000 \mathrm{tr} / \mathrm{min}$. Après cinq tours de roue, soit 35 périodes de l'écoulement, nous avons obtenu une hauteur d'élévation quasi-périodique présentant une valeur moyenne de $78,48 \mathrm{~m}$.

A partir de cette position nous avons réalisé le couplage pompe-circuit et continué en parallèle les calculs couplés et le calcul de la pompe seule.

\section{- 3.2 Calcul de la pompe avec les deux circuits}

Les résultats présentés ci-dessous sont obtenus après convergence, c'est-à-dire lorsque le débit dans la pompe et la hauteur d'élévation présentent un caractère quasi-périodique, soit après une dizaine de révolutions de la roue à partir du couplage des deux codes. Notons que ces simulations nécessitent un temps de calcul très important, sept à dix jours pour une rotation complète de la roue sur une station de travail HP-9000.

\section{NÉCESSITÉ DU CALCUL COUPLÉ}

Dans un premier temps, les résultats numériques montrent la nécessité de réaliser un calcul couplé pompe-circuit afin de connaître précisément le champ de pression dans la pompe. 
En effet, la figure 5 illustre les fluctuations de hauteur d'élévation de la pompe pour deux configurations (en pointillé le cas du calcul de la pompe seule et en trait plein celui de la pompe avec le circuit de $8,57 \mathrm{~m}$ ) en fonction de la position des aubes de la roue par rapport au bec de volute. Pour les deux cas considérés, la hauteur d'élévation présente un minimum relatif lorsqu'une aube se trouve face au bec (abscisse entière) conformément aux travaux expérimentaux de Talha [14] et de Chatel [15] concernant la pompe SHF-A0 (vitesse spécifique $N_{s}$ de 36 , jeu entre la roue et le bec de volute de $3 \%$, roue de 7 aubes).

Cependant, les amplitudes de ces fluctuations pour la fréquence de passage des aubes sont 10 fois plus importantes pour le calcul seul que pour le calcul couplé avec le circuit de $8,57 \mathrm{~m}$ (figure 5.2).

Or Talha [14] a mesuré des amplitudes de l'ordre de $0,7 \%$ pour la fréquence de passage des aubes, ordre de grandeur qui correspond à celui obtenu pour les calculs couplés (figure 9.2).

De plus, l'étude des fluctuations de pression dans la volute (fig. 6 et 7) montre que lorsque le calcul est non couplé, les fluctuations de pression supérieures à $5 \%$ dans la volute se trouvent localisées sur une région très importante $\left(120^{\circ}\right.$ après le bec dans le sens de l'écoulement moyen) alors que pour le calcul couplé cette région est fortement diminuée $\left(<90^{\circ}\right)$ conformément aux observations de Tourret et al. [16] (pour une pompe de 5 aubes, de vitesse spécifique $N_{s}$ de 32 , de jeu entre la roue et le bec de volute de $7 \%$ ).

Nous voyons donc qu'il importe de prendre en compte le circuit environnant afin d'obtenir des fluctuations de pression cohérentes et conformes aux observations expérimentales.

\section{ANALYSE DU COUPLAGE POMPE- CIRCUIT}

\subsection{Débit et hauteur d'élévation de la pompe pour deux configurations de circuits}

Nous venons de montrer que pour le calcul non couplé, les fluctuations de pression sont surestimées par rapport à celles

couramment observées. Nous observons (fig. 8) que quel que soit le circuit, lorsqu'une aube se trouve en face du bec de volute (abscisse entière), le débit dans la pompe diminue. En effet le canal inter-aubes précédant cette aube voit sa section de sortie obstruée par le bec, ce qui entraîne une augmentation de pression au voisinage du bec et une diminution du débit transitant dans le canal ; c'est ce même blocage de débit $[2,7,8]$ que nous observons sur la figure 8.1 . De plus, la répartition du débit dans les canaux est fortement dissymétrique, c'est-à-dire que les canaux non obstrués par le bec de volute voient une augmentation de débit, augmentation entraînant des vitesses en sortie de roue plus importantes d'où une diminution de la pression conformément à l'équation de Bernoulli [8] (fig. 9).

Il est donc nécessaire, lors des études numériques de pompes centrifuges seules, de fixer un débit variable en entrée de roue. Dans le cas contraire, un débit constant en entrée de pompe entraînera une augmentation trop élevée de débit dans les canaux non obstrués par le bec, augmentation qui aura pour conséquence une chute importante de la hauteur d'élévation (fig. 5).

A titre de comparaison, considérons les travaux de Talha [14] qui a obtenu expérimentalement pour la pompe SHF-A0 des fluctuations de débit de $0,10 \%$ en entrée de pompe et de $0,20 \%$ en sortie suivant la fréquence de passage des aubes. Pour nos calculs couplés, nous obtenons le même ordre de grandeur ( $t a b l .2)$ à ceci près que les débits entrant et sortant de la pompe sont identiques (calcul incompressible pour la pompe). De plus, notons que la chute et l'augmentation de
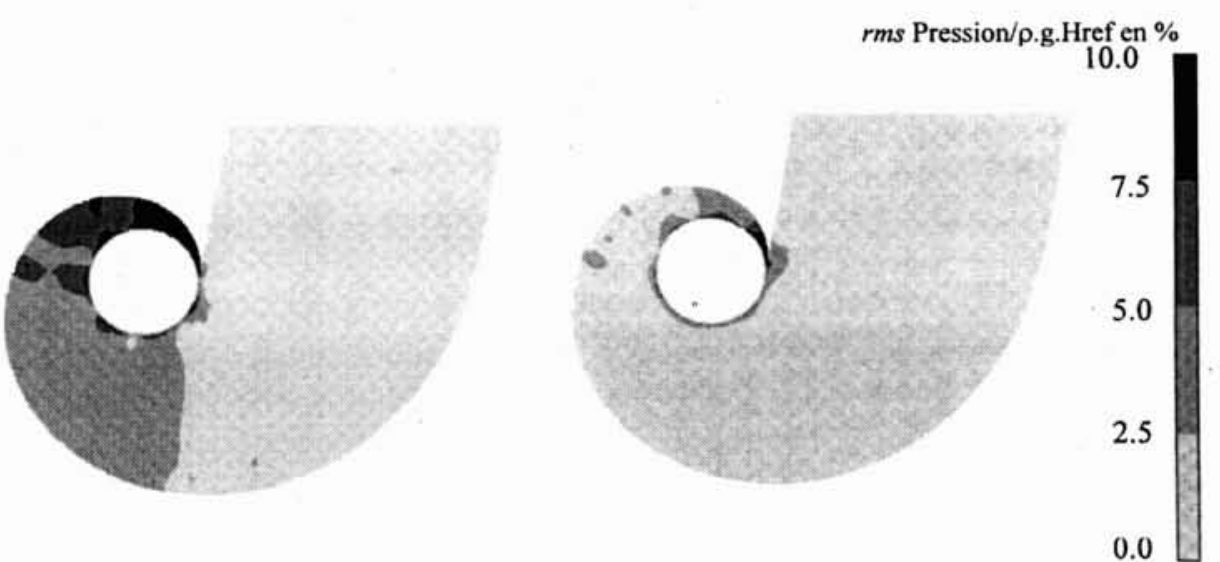

6. Fluctuations de pression dans la volute. 6.1. sans couplage

7. Fluctuations de pression d'après Tourret et $a l$. [16]. 


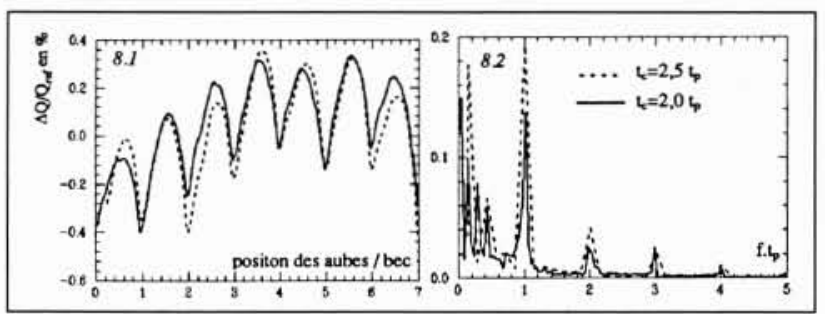

8. Fluctuation du débit.

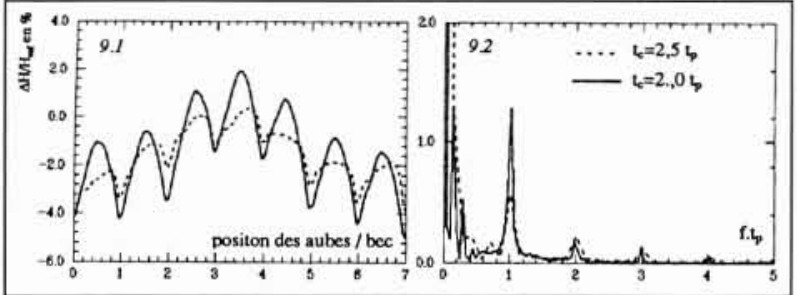

9. Fluctuation de la hauteur d'élévation.

9.1. analyse temporelle 9.2. analyse fréquentielle (Fourier) influence du circuit. influence du circuit.

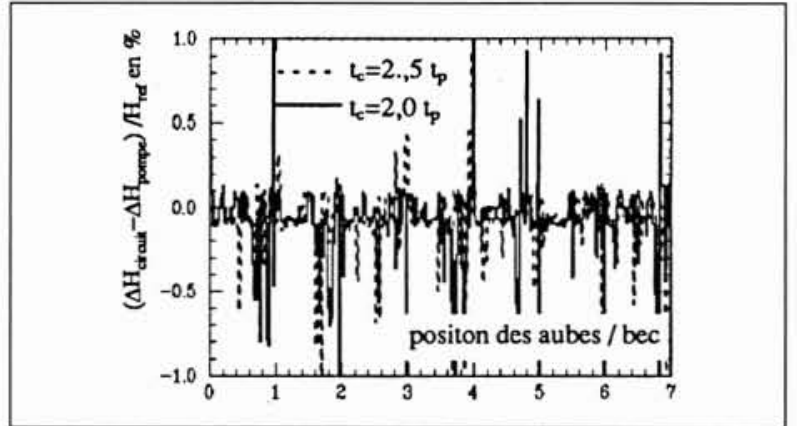

10. Erreur de couplage.

débit se font de manière abrupte (fig. 8.1) et entraînent de fortes oscillations au niveau du couplage des deux codes ; nous n'avons pu obtenir une erreur de convergence en deçà de $1 \%$ (fig. 10) lors du passage des aubes face au bec.

En outre, les fluctuations de débit et de pression varient en fonction de la longueur du circuit considéré. En effet, pour le circuit de $8,57 \mathrm{~m}$ les fluctuations de débit sont légèrement plus importantes que pour le circuit de $10,71 \mathrm{~m}$ où les fluctuations de pression sont plus grandes (fig. $8 \mathrm{et} 9 \mathrm{et}$ tabl. 2).

\subsection{Influence de la longueur du circuit}

Afin d'apporter une réponse à la remarque précédente, les courbes de résonance en débit et en pression sont présentées pour les deux circuits (fig. 11). L'ordonnée de ces courbes représente les fluctuations de débit $\left(Q_{K}\right)$ ou de hauteur piézométrique $\left(H_{k}\right)$ en chaque point $(K)$ du circuit par rapport à la valeur moyenne de ces grandeurs en ces mêmes points $\left(Q_{K \text { moy }}\right.$ et $\left.H_{K \text { moy }}\right)$. La pompe est située à l'abscisse 0,0 (l'aspiration à gauche et le refoulement à droite) et les distances sont rapportées à la longueur d'onde calculée à partir du temps de passage des aubes $t_{p}$. Nous vérifions que le point du circuit à mi-distance de l'aspiration et du refoulement est un naud de pression quelle que soit la longueur du circuit et que la pompe se trouve soit sur un naud de pression pour le circuit de $8,57 \mathrm{~m}$ (puisque $t_{c}=2,0 \mathrm{t}_{\mathrm{p}}$ ), soit sur un ventre pour le circuit de $10,71 \mathrm{~m}$ (puisque $t_{c}=2,5 t_{p}$ ); et inversement pour les nœuds et ventres de débit. En d'autres termes, pour le circuit de $8,57 \mathrm{~m}$, la pompe se trouvant sur un nœud de pression et par conséquent sur un ventre de débit voit ses fluctuations de débit entretenues et celles de pression amorties ; pour le circuit de $10,71 \mathrm{~m}$ nous obtenons le comportement inverse entre la pression et le débit.

Tableau 2. - Amplitudes des fluctuations pour la période de passage des aubes $t_{p}$ (obtenues à partir d'une transformée de Fourier).

\begin{tabular}{|c|c|c|c|c|}
\cline { 2 - 4 } \multicolumn{1}{c|}{} & $\begin{array}{c}\text { Résultats expérimentaux } \\
\text { Talha }[14]\end{array}$ & $\begin{array}{c}\text { Calcul } \\
\text { non couplé }\end{array}$ & $\begin{array}{c}\text { Pompe sur un nœud } \\
\text { de pression } \\
(\mathrm{L}=8,57 \mathrm{~m})\end{array}$ & $\begin{array}{c}\text { Pompe sur un ventre } \\
\text { de pression } \\
(\mathrm{L}=10,71 \mathrm{~m})\end{array}$ \\
\hline $\begin{array}{c}\text { amplitude des fluctuations } \\
\text { de la hauteur d'élévation }\end{array}$ & $0,70 \%$ & $5,00 \%$ & $0,55 \%$ & $1,29 \%$ \\
\hline $\begin{array}{c}\text { amplitude des fluctuations } \\
\text { du débit }\end{array}$ & $\begin{array}{c}0,10 \% \text { en entrée } \\
0,20 \% \text { en sortie }\end{array}$ & $0,00 \%$ & $0,20 \%$ & $0,13 \%$ \\
\hline
\end{tabular}




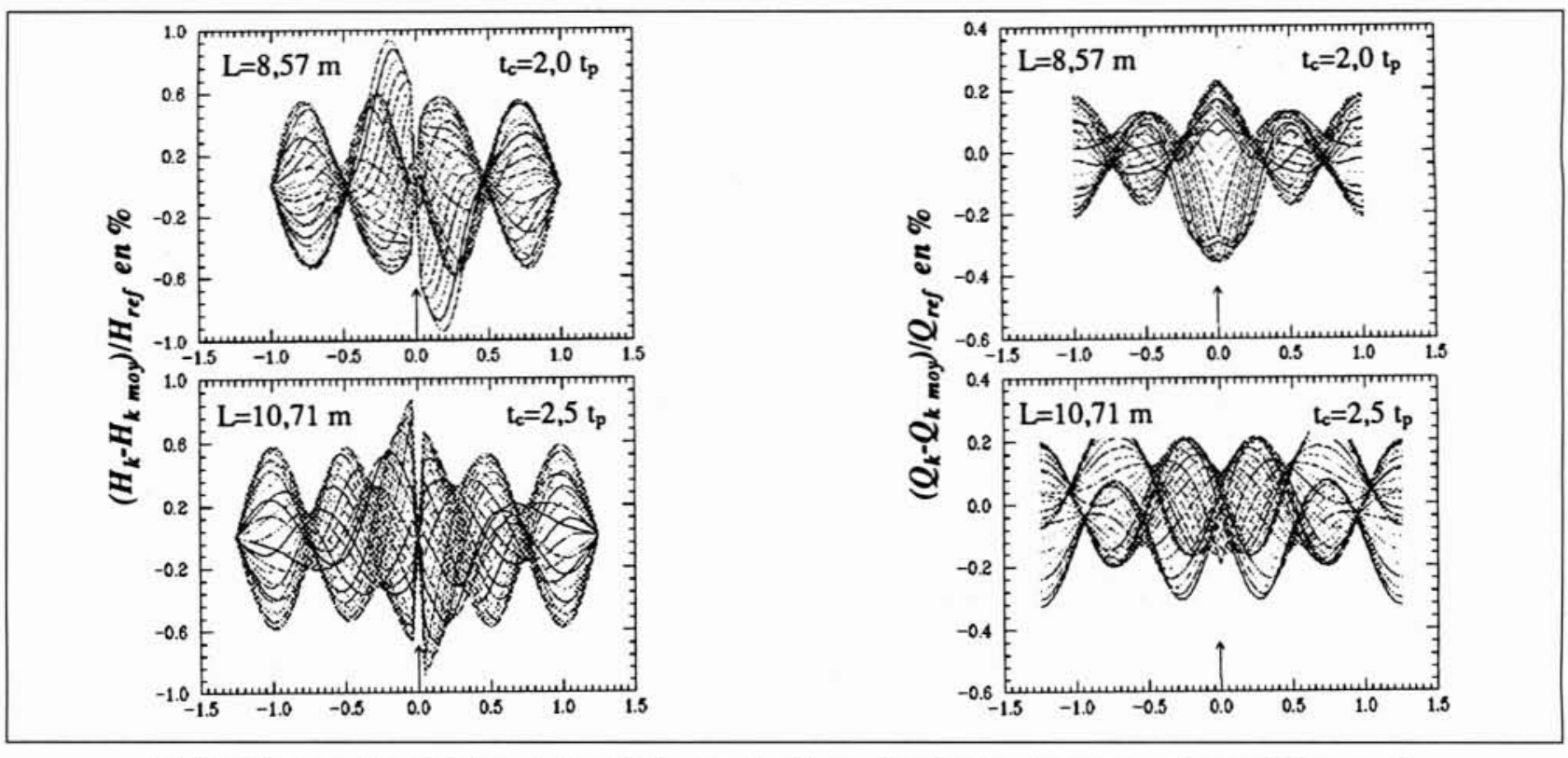

11. Fluctuation de pression et de débit pour les deux circuits (les longueurs des conduites sont adimensionnalisées par la longueur d'onde associée au temps $t_{p}$ ).

\section{VI $\square$ CONCLUSION ET PERSPECTIVES}

Cette étude numérique du couplage pompe-circuit apporte des informations importantes, non seulement sur le fonctionnement de la pompe, mais aussi sur les phénomènes d'interaction pouvant exister.

En effet, lors d'études numériques de pompes centrifuges seules en fluide incompressible, nous avons montré qu'il est nécessaire d'imposer un débit fluctuant comme condition d'entrée afin d'éviter des fluctuations de pression trop importantes par rapport aux études expérimentales : le débit est minimal lorsqu'une aube se trouve face au bec de volute. En outre, ces fluctuations de débit dépendent du circuit et notamment de sa longueur.

Cependant, il reste de nombreux points à étudier et à améliorer.

D'une part, il faudrait vérifier l'influence de certains paramètres géométriques de la pompe, par exemple le jeu entre le bec de volute et le bord de fuite des aubes et montrer ainsi que l'augmentation de ce jeu entraîne une diminution des fluctuations de débit et de pression. L'influence du nombre d'aubes reste également à envisager.

D'autre part, il serait tout aussi intéressant d'étudier d'autres géométries de circuit, comme celle d'un circuit ouvert non symétrique.

Enfin une étude d'un cas réel ayant fait l'objet de mesures en débit et en pression, permettrait de valider la méthode présentée ci-dessus.

\section{BIBLIOGRAPHIE}

[1] Miner S.M., Beaudoin R.J., Flack R.D. (1989). — « Laser velocimeter measurements in a centrifugal flow pump $"$. Journal of Turbomachinery, Vol. 111, pp. 205-212.

[2] Dong R., ChU S., Katz J. (1992), - «Quantitative visualization of the flow within the volute of a centrifugal pump. Part B : Results and analysis ». Journal of Fluids Engineering, Vol. 114, pp. 396-403.

[3] Arndt N., Acosta A.J., Brennen C.E., Caughey T.K. (1990). - « Experimental investigation of rotor-stator interaction in a centrifugal pump with several vaned diffusers $»$. Journal of Turbomachinery, Vol. 112, pp. 98-108.
[4] Miner S.M., Flack R.D., Allaire P.E. (1992). - « TwoDimensional flow analysis of a laboratory centrifugal pump ». Journal of Turbomachinery, Vol. 114, pp. 333-339.

[5] ChaprN V. (1993). - «Contribution à la simulation numérique de l'interaction aérodynamique de deux roues ailetées » Thèse de Doctorat, Université Pierre et Marie Curie.

[6] Fatsis A. (1993). - « Three dimensional unsteady flow calculations in radial components ". Proceedings Spacecraft Propulsion, von Karman Institut for Fluid Dynamics, Lecture séries 1993-01, Belgium.

[7] Fortes Patella R., Longatte F., Kueny J.-L., Croba D. (1995). — « Numerical analysis of unsteady flow in a centrifugal pump ». Proceedings of ASME Fluid Machinery Forum, Head Island, pp. 41-46.

[8] Longatte F. (1995). - « Analyse phénoménologique du couplage rotor-stator». DEA, Institut National Polytechnique de Grenoble.

[9] HuREAu F. (1989), — « Méthode théorique et expérimentale de caractérisation des écoulements instationnaires dans les pompes centrifuges ». Thèse de Doctorat, Université de Nantes.

[10] BERT P.F. (1996). — « Modélisation des écoulements instationnaires dans les turbomachines par une méthode éléments finis ". Thèse de Doctorat, Institut National Polytechnique de Grenoble.

[11] Cıoc D. (1963). — « Sistematizarea algoritmilor pentru calculul loviturii de berbec ». Studii de Hidraulica, XI, ISCH, Bucuresti.

[12] Ubaldi M., Zunino P., Barigozzi G., Cattanei A. (1996). - «An experimental investigation of stator induced unsteadiness on centrifugal impeller outflow ». Journal of Turbomachinery, Vol. 118, pp. 41-51.

[13] Sedille M. (1967). - « Turbomachines hydrauliques et thermiques ». tome 2, Masson et Cie, Paris.

[14] Talha A. (1996). - « Etude hydroacoustique d'une pompe centrifuge et de son circuit immédiat par l'analyse expérimentale des pressions et des vitesses instationnaires ». Thèse de Doctorat, Université des Sciences et Technologies de Lille.

[15] Chatel G. (1993). - « Caractérisation des sources de bruit dans une pompe ». EDF-DER et SECAV, Rapport 930820, Fascicule 2.

[16] Tourret J., Badie-Cassagnet A., Bernard G. Foucault J.-P. (1985). - « Etude expérimentale du bruit généré et émis par une pompe centrifuge ». La Houille Blanche, $n^{\circ} 8$, pp. 663-676. 